\title{
Auto-regressive moving average analysis of linear and discontinuous models of human balance during quiet standing
}

\author{
Piotr Kowalczyk, ${ }^{1}$ Salam Nema, ${ }^{2}$ Paul Glendinning, ${ }^{3}$ Ian Loram, ${ }^{4}$ and Martin Brown ${ }^{5}$ \\ 1) School of Computing, Mathematics and Digital Technology, Manchester Metropolitan University, Chester Street, \\ Manchester, M1 5GD, U.K.,E-mail: p.kowalczyk@mmu.ac.uk \\ 2) School of Computing, Mathematics and Digital Technology, Manchester Metropolitan University, Chester Street, \\ Manchester, M1 5GD, U.K.,E-mail: s.nema@mmu.ac.uk \\ ${ }^{3)}$ School of Mathematics and Centre for Interdisciplinary Computational and Dynamical Analysis (CICADA), \\ University of Manchester, Manchester, M13 9PL,U.K.,E-mail: paul.glendinning@manchester.ac.uk \\ ${ }^{4)}$ Institute for Biomedical Research into Human Movement and Health (IRM), \\ Manchester Metropolitan University,Chester Street, Manchester, M1 5GD, U.K., \\ E-mail: i.loram@mmu.ac.uk \\ ${ }^{5)}$ School of Electrical and Electronic Engineering, The University of Manchester Manchester, M13 9PL, U.K., \\ E-mail: Martin.Brown@manchester.ac.uk
}

Linear Time Invariant (LTI) processes can be modelled by means of Auto-Regressive Moving Average (ARMA) model systems. In this paper, we examine whether an ARMA model can be fitted to a process characterised by switched nonlinearities. In particular, we conduct the following test: we generate data from known LTI and nonlinear (threshold/dead-zone) models of human balance and analyse the output using ARMA. We show that both these known systems can be fitted, according to standard criteria, with low order ARMA models. To check if there are some obvious effects of the dead-zone, we compare the power spectra of both systems with the power spectra of their ARMA models. We then examine spectral properties of three posturographic data sets and their ARMA models and compare them with the power spectra of our model systems. Finally, we examine the dynamics of our model systems in the absence of noise to determine what is the effect of the switching threshold (dead-zone) on the asymptotic dynamics.

keywords: linear stochastic model, switched model, ARMA fitting, posturographic data.

There is an ongoing controversy, among the biomechanics community, whether the neuromuscular system which controls human posture during quiet standing can be better described as being linear, continuous and time invariant (LTI), or whether an intermittent (switched/containing dead-zones) control captures better the neuromuscular control. Fitting the experimental data to models which are LTI (for instance to Auto Regressive Moving Average Models ARMA) have been used to imply that the process, which generates the time series data to which an LTI model is fitted, is linear and time invariant. In presented work, we show, by means of two representative explicit examples, that ARMA models can be successfully fitted to models of human balance which are linear as well as to models which contain discontinuities (a dead-zone). Moreover, if data generated from these models are compared with real posturographic data it is impossible to judge (without some in-depth and detailed analysis of the ARMA models) which of the two explicit models describes better the posturographic data. Hence, successful linear model fitting (e.g. ARMA) may lead to over interpretation of the results.

\section{INTRODUCTION}

Although investigations of human balance during quiet standing have a long history, see for instance ${ }^{1-6}$, the fundamental question related to the character of the motor control during quiet standing remains an open issue. It is often assumed that the motor control, which ensures balance during quiet standing of humans, can be modelled using linear, continuous time, feedback models $^{7-11}$. Such models exclude nonlinearites, e.g. thresholds, and time variant processes, e.g. open loops. Thresholds can create complex dynamics such as periodic oscillations $^{12}$ or micro-chaotic behaviour ${ }^{13}$. In the presence of multiplicative noise, it is was suggested in ${ }^{14}$ that the presence of thresholds implies different power laws in correlation function.

One of the strategies, to fit a linear model to a times series posturographic data, is to use ARMA modelling ${ }^{2,7,8}$. To apply ARMA fitting one assumes that time series data is generated by a process which is stationary. Stationarity, in turn, implies that the process is time invariant and without transient dynamics. Often adding more dimensions to an ARMA model improves a data fit. However, it well may be that the process could be understood better by a nonlinear time variant model, e.g. a switched model with a threshold. In fact, it has been recently suggested that control mechanisms such as intermittent controllers ${ }^{16,17}$, which naturally lead to nonlinear models, may also be used and may provide even better explanation for the 
characteristic features of the posturographic time series data of human quiet standing. Using as a discriminant the two classes of controllers, we can broadly divide mathematical models of quiet standing into a class of (a) linear stochastic models ${ }^{7,8,10,18}$ and (b) nonlinear models (with, for instance, thresholds or time based open loops in the feedback control) ${ }^{12,15-17,19-22}$.

The main aim of the paper is to investigate, by means of a known LTI and a nonlinear system, both of which are models of quiet standing, whether a time series data that contains nonlinearities can be consistently fitted by means of ARMA. The two model systems can be thought of as 'experimental black boxes' which are fully known to us. We will verify numerically if it is possible to successfully fit ARMA models to time series data generated from both model system. We will then compare the ARMA models of our systems with ARMA models of experimental data.

$\mathrm{In}^{28,29}$, it has been shown that noise has a linearizing effect on systems with thresholds provided that its properties are appropriately tuned. Thus, not to induce this effect deliberately which would make our experiment superfluous, we use additive Gaussian noise without choosing the intensity of the noise to obtain this linearizing response. We will then seek to find some signatures of ARMA (e.g. a consistent significant difference in the order of ARMA models) which could be used as an indication that a time series was produced by a nonlinear or a time variant system. To verify the ARMA fit we compare the power spectra of our ARMA models with the power spectra of the signals used to produce the models. We also compare the power spectra of system generated ARMA models with the power spectra of the posturographic ARMA model systems to see how well the ARMA models describe the posturographic data.

The rest of the paper is outlined as follows. In Sec. II, we link an ARMA model with its continuous time representation. We highlight that it is a power spectrum of a signal which is aimed to be fitted by means of ARMA. Then, in Sec. III and IV our continuous time model systems - the linear and switched one, which we use to produce the data sets - are introduced, and the results of ARMA model fitting are presented. In Sec. V, we make a comparison between the power spectra of system generated ARMA models and experimentally derived ARMA model systems. Finally, in Sec. VI we conclude the paper and highlight the directions of future research.

\section{ARMA MODELS: DESCRIPTION}

To understand the numerical experiments which we conduct in the main part of the paper, we present an interpretation of ARMA model systems, of some order $(p, q)$, as continuous time systems.

\section{A. ARMA as a differential equation}

The ARMA model of order $(p, q)$ is

$y_{n}=\alpha_{1} y_{n-1}+\cdots+\alpha_{p} y_{n-p}+\varepsilon_{n}-\beta_{1} \varepsilon_{n-1}-\cdots-\beta_{q} \varepsilon_{n-q}$,

where $\alpha_{1}, \alpha_{2}, \cdots, \alpha_{p}$ are the autoregressive and $\beta_{1}, \beta_{2}, \cdots, \beta_{q}$ are the moving average coefficients; $\varepsilon$ is white noise process. The aim of this section is to describe an explicit (stochastic) ordinary differential equation with a solution at times $n \Delta t$ equal to the solution of the ARMA difference equation (1) at time step $n$. Here $\Delta t$ represents the time step at which samples of data are taken and used to construct the ARMA model. Note that we can consider the random variables $\left(\varepsilon_{j}\right)_{j=-q}^{\infty}$ as a given (fixed) sequence in our construction of a model equation. The differential equation derived later serves as the continuous-time descriptive model equivalent to a discrete time ARMA model of some order $(p, q)$.

By setting

$$
z_{n}^{(1)}=y_{n-p}, \quad z_{n}^{(2)}=y_{n-p+1}, \quad \ldots, \quad z_{n}^{(p)}=y_{n-1},
$$

the difference equation (1) can be written as a $p$ dimensional difference equation for the vector $z_{n}=$ $\left(z_{n}^{(1)}, \ldots, z_{n}^{(p)}\right)$. Namely

$$
z_{n}=A z_{n-1}+b_{n}
$$

where the $p \times p$ matrix $A$ and the vectors $b_{n} \in B R^{p}$ are given by

$$
A=\left(\begin{array}{ccccc}
0 & 1 & 0 & \ldots & 0 \\
0 & 0 & 1 & \ldots & 0 \\
\vdots & \vdots & \vdots & & \vdots \\
0 & 0 & 0 & \ldots & 1 \\
\alpha_{p} & \alpha_{p-1} & \alpha_{p-2} & \ldots & \alpha_{1}
\end{array}\right), \quad b_{n}=\left(\begin{array}{c}
0 \\
0 \\
\vdots \\
0 \\
\xi_{n}
\end{array}\right)
$$

where $\xi_{n}$ is a real random variable derived from the $\varepsilon_{t}$ via

$$
\xi_{n}=\varepsilon_{n}-\beta_{1} \varepsilon_{n-1}-\cdots-\beta_{q} \varepsilon_{n-q} .
$$

If (2) is considered as an initial value problem given some initial $z_{0}$ then a straightforward induction argument shows that

$$
z_{n}=A^{n} z_{0}+\left(b_{n}+A b_{n-1}+\cdots+A^{n-1} b_{1}\right) .
$$

Our aim now is to write down a differential equation for a $p$-dimensional variable $y$ such that the solution $y(t)$ satisfies

$$
y(n \Delta t)=z_{n}
$$

for all $n \geq 0$. Note that this is not the same as finding a differential equation for which the ARMA difference equation (1) is a numerical approximation scheme. We actually seek strict equality (there will be 
a small technical issue in the answer: equality will hold only in the limit as $t \downarrow n \Delta t$, see below).

The key observation is that if $u(t)$ is a given integrable function then the solution of the $p$-dimensional differential equation

$$
\dot{y}=C y+u
$$

where $C$ is a constant $p \times p$ matrix, is

$$
y(t)=e^{t C} y(0)+\int_{0}^{t} e^{(t-s) C} u(s) d s .
$$

This is a standard equation obtained by multiplying (6) through by the integrating factor $e^{-t C}$ and then integrating. For the particular choice of

$$
u(t)=\sum_{1}^{\infty} b_{n} \delta(t-n \Delta t) \quad \text { for } \quad t>0
$$

the integral in (7) can be calculated explicitly. Let $r_{+}$ denote the limit as $t$ tends to $r$ from above. Then for $n>0$ the solution at $n \Delta t_{+}$is obtained by integrating from $0_{+}$to $n \Delta t_{+}$in $(7)$ giving

$$
y\left(n \Delta t_{+}\right)=e^{n \Delta t C} y\left(0_{+}\right)+\sum_{1}^{n} e^{(n \Delta t-k \Delta t) C} b_{k}
$$

The similarity with (5) is perhaps clearer if this is rewritten as

$y\left(n \Delta t_{+}\right)=\left(e^{\Delta t C}\right)^{n} y\left(0_{+}\right)+\left(e^{n \Delta t C}\right)^{n-1} b_{1}+\left(e^{n \Delta t C}\right)^{n-2} b_{2}+b_{n}$.

which is, of course, precisely the solution (5) if

$$
e^{\Delta t C}=A
$$

In our problem the matrix $A$ is known and given by (3), as is the sampling time $\Delta t$, so (11) is an implicit equation for a matrix $C$ in terms of a real constant $\Delta t$ and a given real matrix $A$. Solving for $C$ is not entirely trivial as this means taking the logarithm of a matrix.

We are interested in $(2, q)$-ARMA models with complex eigenvalues to the deterministic part and so we restrict attention to this case below; second order models have been suggested as providing a good fit for the time series data of quiet standing of humans, see for instance ${ }^{8}$.

Note that we may assume, by a small perturbation if necessary, that $A$ has simple eigenvalues and hence that it is diagonalizable. The standard theory of matrix logarithms then shows that in the general $n$-dimensional case the model differential equation is also $n$-dimensional unless some of the eigenvalues of $A$ are negative, in which case the dimension is higher than $n$ as $C$ is necessarily complex.

In the two-dimensional case we are interested in

$$
A=\left(\begin{array}{cc}
0 & 1 \\
\alpha_{2} & \alpha_{1}
\end{array}\right)
$$

with $\alpha_{2}<0$. If $\alpha_{1}^{2}<-4 \alpha_{2}$ then the eigenvalues of $A$ are complex: $\lambda_{1}=r e^{-i \theta}$ and $\lambda_{2}=\lambda_{1}^{*}$, with $0<\theta<\pi$. It is a simple computation to show that if $E$ is the matrix of eigenvectors,

$$
E=\left(\begin{array}{cc}
1 & 1 \\
\lambda_{1} & \lambda_{2}
\end{array}\right)
$$

then

$$
A E=E \Lambda, \quad \Lambda=\operatorname{diag}\left(\lambda_{1}, \lambda_{2}\right) .
$$

Therefore $E^{-1} A E=\Lambda$ and by (11) $E^{-1} e^{\Delta t C} E=\Lambda$ from which we can read off one solution (the principal logarithm) for $\Delta t C$ :

$$
\Delta t C=E\left(\begin{array}{cc}
\sigma_{1} & 0 \\
0 & \sigma_{2}
\end{array}\right) E^{-1}
$$

where

$$
\sigma_{1}=\ell n r-i \theta, \quad \text { and } \quad \sigma_{2}=\ell n r+i \theta .
$$

Now writing (15) out in full gives

$$
n \Delta t C=\frac{1}{\lambda_{2}-\lambda_{1}}\left(\begin{array}{cc}
1 & 1 \\
\lambda_{1} & \lambda_{2}
\end{array}\right)\left(\begin{array}{cc}
\sigma_{1} & 0 \\
0 & \sigma_{2}
\end{array}\right)\left(\begin{array}{cc}
\lambda_{2} & -1 \\
-\lambda_{1} & 1
\end{array}\right)
$$

and so multiplying this out explicitly, the obtained equation can be written as:

$$
\Delta t C=\frac{1}{\lambda_{2}-\lambda_{1}}\left(\begin{array}{cc}
\lambda_{2} \sigma_{1}-\lambda_{1} \sigma_{2} & \sigma_{2}-\sigma_{1} \\
-\lambda_{1} \lambda_{2}\left(\sigma_{2}-\sigma_{1}\right) & \lambda_{2} \sigma_{2}-\lambda_{1} \sigma_{1}
\end{array}\right) .
$$

Although this looks messy, explicit evaluation using (16) gives a series of cancellations leaving the result

$$
\Delta t C=\left(\begin{array}{cc}
\ell n r-\theta \cot \theta & \theta /(r \sin \theta) \\
-r \theta /(\sin \theta) & \ln r+\theta \cot \theta
\end{array}\right)
$$

which is, of course, a real matrix.

We can now write the continuous time equation (6) that models the ARMA equation of order $(2, q)$, which is a forced linear second order differential equation with constant coefficients. However, for our purposes it is sufficient to analyse the matrix $C$. The eigenvalues of $C$ are

$$
\mu_{1}=\frac{1}{\Delta t}(\ln (r)+i \theta) \quad \text { and } \quad \mu_{2}=\frac{1}{\Delta t}(\ln (r)-i \theta) .
$$

Thus, the natural timescale of motion is of order $\Delta t$; indeed, $\Delta t$ could be eliminated by working in a rescaled time $\Delta t^{-1} t$. Now, if $r<1$ (corresponding to damped linear motion in the ARMA model $)$ then $\ln (r)<0$ and so the system is damped, but also the oscillatory part of the solution has frequency equal to $\theta / \Delta t$. By deriving the second order system from the set of first order equations (6), we can see that the forcing term is a weighted sum of Dirac delta distributions (the derivative of a Dirac delta is a sum of delta distributions) based on the noise input of ARMA over more than one time. 


\section{B. ARMA - stochastic description}

To apply ARMA models, it is assumed that the time series which is modelled is produced by a stationary process, which implies that the mean and variance of the process are constant over time, and the autocovariances depend only on the time shift, say $\Delta t$, for all $\Delta t$. Considering $\left(y_{1}, y_{2}, y_{3}, \ldots, y_{m}\right)$ to be a sequence of observations at times $\left(t_{1}, t_{2}, t_{3}, \ldots, t_{m}\right)$ of a stationary process, we have

$$
\begin{aligned}
& C_{y_{1}, y_{1+\Delta t}}=C_{y_{2}, y_{2+\Delta t}}=\ldots=C_{y_{m-\Delta t}, y_{m}}=\gamma_{1}, \\
& C_{y_{1}, y_{1+2 \Delta t}}=C_{y_{2}, y_{2+2 \Delta t}}=\ldots=C_{y_{m-2 \Delta t}, y_{m}}=\gamma_{2} \text {, } \\
& C_{y_{1}, y_{1+(m-1) \Delta t}}=\gamma_{m-1}
\end{aligned}
$$

where $C$ is the autocovariance

$$
C_{y_{t}, y_{t+\Delta t}}=E\left[\left(y_{t}-\mu\right)\left(y_{t+\Delta t}-\mu\right)\right]
$$

with $E$ and $\mu$ denoting the expected value and the mean of the process respectively.

The modelling of a times series is then reduced to the estimation of a constant mean, say $\mu=C$, and an autocovariance matrix derived from the $m$ observations. In the process of ARMA modelling, it is the autocovariance function coefficients $\gamma_{i} / \gamma_{0}(i=$ $1, \ldots, m-1)$, where $\gamma_{0}$ is the variance of the process, with their characteristic decay, that indicate the order of an ARMA process. Since the Fourier transform of the autocovariance function gives power spectral density then ARMA modelling is aimed at fitting power spectra corresponding to a time series that is modelled. To be precise, it is the Fourier transform of the autocorrelation function, that is when $\mu=0$ in the autocovariance formula (20), which gives power spectral density of a stochastic signal, by Wiener-KhintchineEinstein Theorem ${ }^{23}$.

\section{CONTINUOUS TIME SYSTEMS}

We will now introduce the continuous time system of interest that we use to conduct part of the numerical investigations which are the subject of the paper. The nonlinear model with a threshold is introduced later in Sec. IV.

To understand the model, consider human subjects standing on both legs with eyes closed or open. We are interested in investigating the sway motion that occurs in the sagittal plane, i.e. we consider a forward-backward body sway. We simplify the biomechanics of the body by representing it as an inverted pendulum with the body sway occurring in the sagittal plane about the ankle joint axis. Gravity $g$ acts on the centre-of-mass when the angle $\phi$ (measured in radians) between the vertical ankle joint axis and the body's position becomes non-zero; when there is no sway the body is vertical and $\phi=0$. The centre of mass $m$ is located at height $h$ above the ankle joint axis. The sway motion will then be modelled by a delay differential equation with additive white noise of the form

$$
J \ddot{\phi}=m g h \sin (\phi)+T+\sigma \zeta(t),
$$

where $J$ is the moment of inertia of the body about the ankle joint axis, $m$ is the mass of the body, $h$ is the distance from the ankle joint axis to the centre of mass and $\zeta(t)$ is Gaussian white noise of intensity $\sigma$. The delay terms are present in the applied torque generated by a Proportional-Derivative controller. Namely

$$
T=-K_{p} \phi(t-\tau)-K_{d} \dot{\phi}(t-\tau),
$$

where $K_{p}$ and $K_{d}$ are negative constants, and $\tau>0$ is the time delay.

We are interested in system dynamics for small values of the angle $\phi$, and approximating $\sin (\phi) \approx \phi$ simplifies the equation (21) to

$$
J \ddot{\phi}=m g h \phi+T+\sigma \zeta(t) .
$$

Expressing (22) as a set of first order equations, by setting $\dot{\phi}=x$, gives

$$
\begin{aligned}
& \dot{\phi}=x \\
& \dot{x}=m g h / J \phi-K_{p} / J \phi(t-\tau)-K_{d} / J x(t-\tau)+\sigma / J \zeta(t)
\end{aligned}
$$

\section{A. Euler's method for systems with time delay and white noise}

System (23) is a linear stochastic system with time delay. The angle $\phi$ and the angular velocity $x=\dot{\phi}$ are now random variables. It has been shown in ${ }^{24,25}$ that stochastic delay differential equations

$$
\dot{x}(t)=f(x(t), x(t-\tau))+\sigma \zeta(t),
$$

where $\tau$ is the time delay, $\zeta(t)$ is Gaussian white noise with intensity $\sigma$, can be approximated by

$$
x_{n+1}=x_{n}+f\left(x_{n}, x_{n-k}\right) h+\sigma W_{n} \sqrt{h},
$$

for $h$ sufficiently small; $\tau$ is the time delay, $h$ is the step size, $k=\tau / h$, and $W_{n}$ is the standard Wiener process. Similarly, a stochastic differential equation

$$
\dot{x}(t)=f(x(t))+\sigma \zeta(t),
$$

can be approximated by a discrete system

$$
x_{n+1}=x_{n}+f\left(x_{n}\right) h+\sigma W_{n} \sqrt{h} .
$$

Above numerical scheme was used to generate the time series trajectory of (23). The standard Wiener process is approximated numerically at each step $t_{n}$ by a function that generates psudo-random numbers with expected value $\mu=\mathbf{E}[X]=0$ and standard deviation $\sigma=$ $\sqrt{\mathbf{E}\left[(X-\mu)^{2}\right]}=1$, where $X$ is a random variable. 


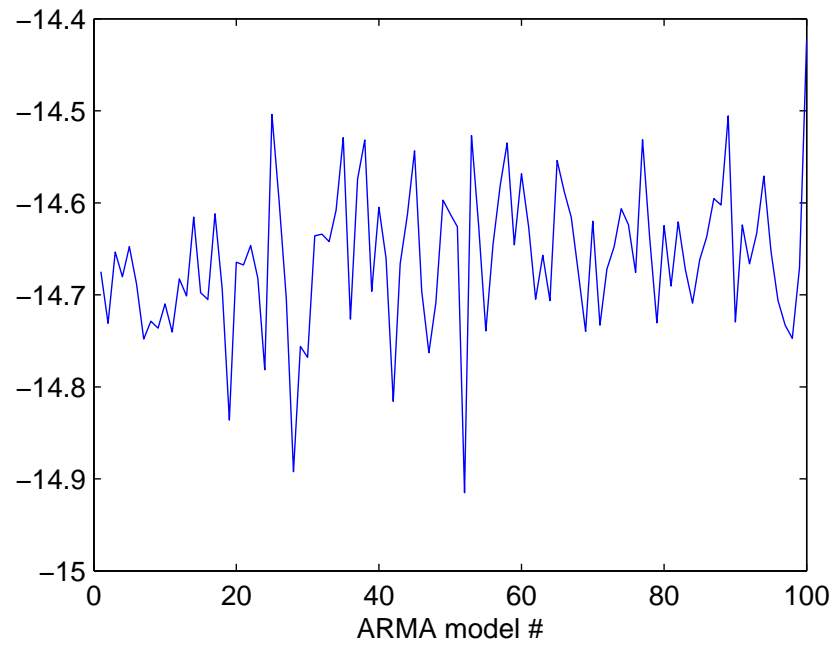

FIG. 1. AIC values for different ARMA models (Continuous system).

\section{B. System dynamics - numerical exploration}

For the following simulations, we take physiologically feasible values, similar to those used in ${ }^{26}$, and we set $m=66 \mathrm{~kg}, h=0.87 \mathrm{~m}, g=9.81 \mathrm{~m} / \mathrm{s}^{2}, J=66 \mathrm{kgm}^{2}$, time delay $\tau=0.15 \mathrm{~s}$, the control coefficients $K_{p}=$ $720 \mathrm{Nm} / \mathrm{rad}$ and $K_{D}=300 \mathrm{Nms} / \mathrm{rad}$, and the noise torque $\sigma=2 \mathrm{Nm}$. Numerical simulations were performed with a fixed step size of $0.001 \mathrm{~s}$ for the duration of $160 \mathrm{~s}$. The data corresponding to first $120 \mathrm{~s}$ were discarded as describing the transient dynamics. The data were then filtered using the low pass butterworth filter with the cutoff frequency of $4 \mathrm{~Hz}$.

\section{ARMA fitting}

To perform ARMA fitting, the data were imported to Matlab systems identification toolbox, which uses non-linear least squares approximation for parameter estimation. In each numerical experiment 402 data points were used for the position and velocity components. One hundred ARMA models were determined from the hundred time series data sets generated from the continuous time model system (23). The Akaike's Information Criterion (AIC) ${ }^{27}$ was used to determine orders $(p, q)$ of the ARMA models. Fig. 1 shows the AIC values for all the ARMA models. The fit of the model is considered to be better the lower the value of the $\mathrm{AIC}^{23}$.

The coefficients $\alpha_{i}(i=1,2)$ and $\beta_{j}(j=1,2)$ listed in Tab. I correspond to the average ARMA model coefficients of order $(2,2)$ obtained from the one hundred ARMA models. Additionally, the standard deviation for each ARMA coefficients set were calculated and
TABLE I. ARMA coefficients (for the linear stochastic system).

\begin{tabular}{lccccc}
\hline \hline & $\alpha_{1}$ & $\alpha_{2}$ & $\beta_{1}$ & $\beta_{2}$ & Fit \% \\
\hline position (average) & 1.5953 & -0.6433 & 0.9722 & 0.3302 & 91.25 \\
\hline velocity (average) & 1.3211 & -0.6129 & 0.1152 & -0.1343 & 56.23 \\
\hline position (single run) & 1.5931 & -0.6410 & 0.9631 & 0.3296 & 91.93 \\
\hline velocity (single run) & 1.3208 & -0.6133 & 0.1125 & -0.1319 & 56.62 \\
\hline Standard deviation & 0.0451 & 0.0437 & 0.0660 & 0.0549 & \\
\hline \hline
\end{tabular}

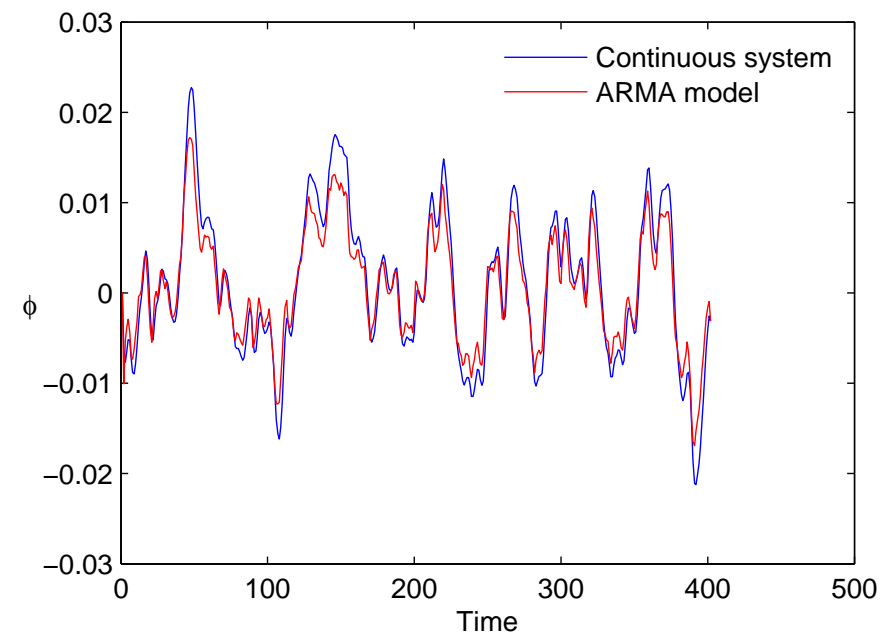

FIG. 2. Comparison between time series of the continuous time system, and its equivalent ARMA model (single run).

presented. It should be noted that, to produce the time series data of an ARMA model using its model equation (1), it is necessary to calculate the variance of white noise which appears in the equation. The standard deviation of white noise was found to be $\sigma=0.000784{\text { ( } \text { see }^{30} \text { for }}$ details on how to compute the standard deviation of white noise of an ARMA model).

We note that better fit was obtained for the position than for the velocity time series data. In the next section, we analyse the time series data produced by the continuous time system (23) and its ARMA equivalent model determined by the coefficients shown in Tab.I. In particular, we use time series data corresponding to the angular position in Tab.I.

\section{Frequency spectrum}

In this section, we will investigate the frequency spectra of representative time series data set produced by our model system (23) and its ARMA discrete time representation given by equation (1). In Fig. 2 we show a comparison between a single realisation of the continuous time system (23) (we consider the position component) and its ARMA equivalent. It can be seen that ARMA model captures the main components of the time series 


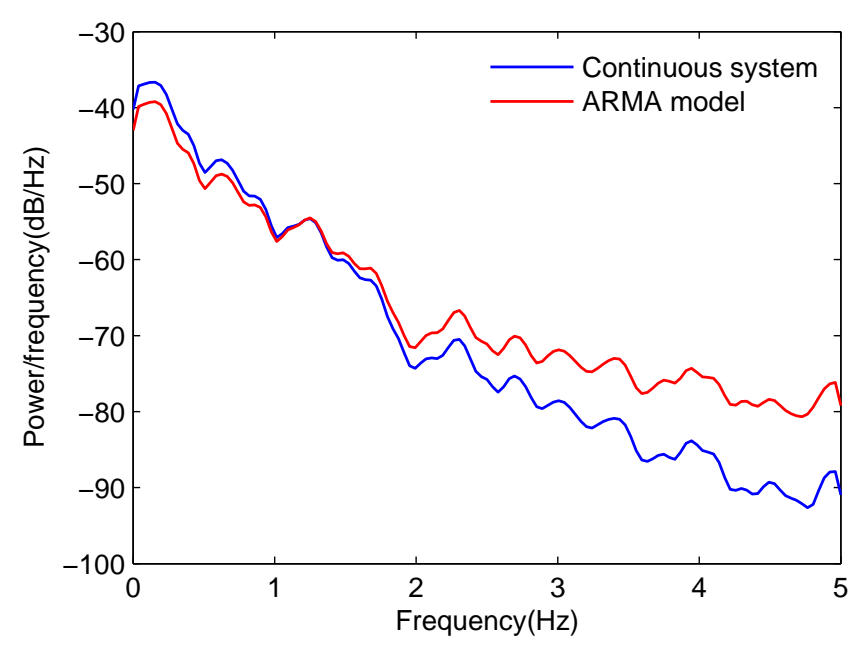

FIG. 3. Welch power spectral density estimate of the continuous time system, and its equivalent ARMA model (single run).

data produced by our continuous time system.

Since ARMA fitting is based on matching the frequency spectra of a signal, in the following we will take a look at the character of the frequency-power spectrum corresponding to the position time series data and its ARMA model. In Fig. 3, we are depicting a comparison between the Welch power spectral density estimate ${ }^{31}$ of the position signals depicted in Fig. 2. We will also take a look at the character of the frequency-power spectrum corresponding to the average position time series data and its ARMA equivalent (see Fig. 4). It can be seen that there is a slight power difference between the models at the low frequency range, while there is a significant power difference at the higher frequency range, which is as expected since we would need a higher degree ARMA model system to capture the higher frequency components. Note that the effect of considering an averaged power spectra is that of smoothing the spectral curves (compare Figures 3 and 4).

To further investigate the ARMA model system and its equivalent continuous time representation given by equation (6), we compute the matrix $C$ which gives the deterministic part of the continuous time representation of the ARMA model. Using equation (19), the matrix $C$ (taking the average values, from Tab. I, of the ARMA position component) can be shown to be equal to

$$
C=\left(\begin{array}{cc}
-12.046 & 12.434 \\
-8.113 & 7.776
\end{array}\right)
$$

We can verify that the eigenvalues of the matrix $C$ are equal to $\lambda_{1}=-2.134+1.627 i$ and $\lambda_{2}=-2.134-$ 1.627i. Thus, our original continuous time system (23) corresponds to a second order linear oscillator with damped oscillatory motion driven by noise.

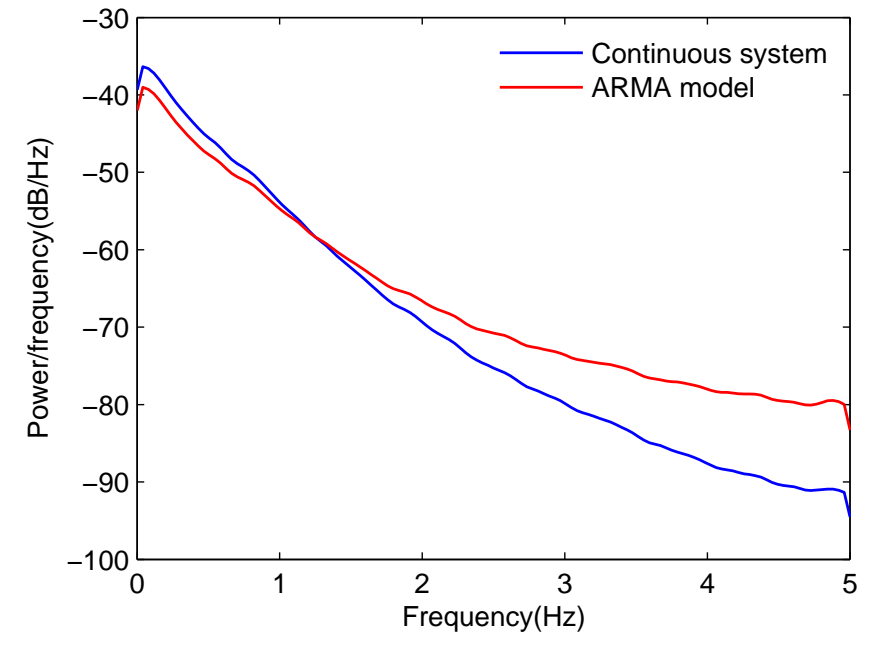

FIG. 4. Averaged power spectral density estimate of the continuous time system, and its equivalent ARMA model.

\section{DISCONTINUOUS SYSTEM}

We now modify the continuous time system (23). We assume that to control the upright position a corrective torque $T$ is applied through a PD controller when some fixed, but non-zero, positive threshold $\phi_{0}$ is detected. This leads to the following model equations:

$$
J \ddot{\phi}=m g h \sin (\phi)+\sigma \zeta(t) \text { for }|\phi(t-\tau)| \leq \phi_{0},
$$

when there is no control applied to the system, and

$$
J \ddot{\phi}=m g h \sin (\phi)+T+\sigma \zeta(t) \text { for }|\phi(t-\tau)|>\phi_{0},
$$

where $T=-K_{p} \phi(t-\tau)-K_{d} \dot{\phi}(t-\tau)$, when there is control applied; the meaning of the parameters is the same as in the continuous time case. Again, making the approximation $\sin \phi \approx \phi$, which is justifiable for small angles $\phi$ of the body sway, the model equations (28) and (29) become

$$
J \ddot{\phi}=m g h \phi+\sigma \zeta(t) \quad \text { for } \quad|\phi(t-\tau)| \leq \phi_{0},
$$

and

$$
J \ddot{\phi}=m g h \phi+T+\sigma \zeta(t) \text { for }|\phi(t-\tau)|>\phi_{0} .
$$

Expressing (30) and (31) as a set of first order equations, by setting $x=\dot{\phi}$, we obtain a switched stochastic system. Namely for $\phi(t-\tau) \leq \phi_{0}$, we have

$$
\begin{aligned}
& \dot{\phi}=x \\
& \dot{x}=m g h / J \phi+\sigma / J \zeta(t)
\end{aligned}
$$

and for $\phi(t-\tau)>\phi_{0}$ we obtain

$$
\begin{aligned}
& \dot{\phi}=x \\
& \dot{x}=m g h / J \phi-K_{p} / J \phi(t-\tau)-K_{d} / J x(t-\tau)+\sigma / J \zeta(t) .
\end{aligned}
$$




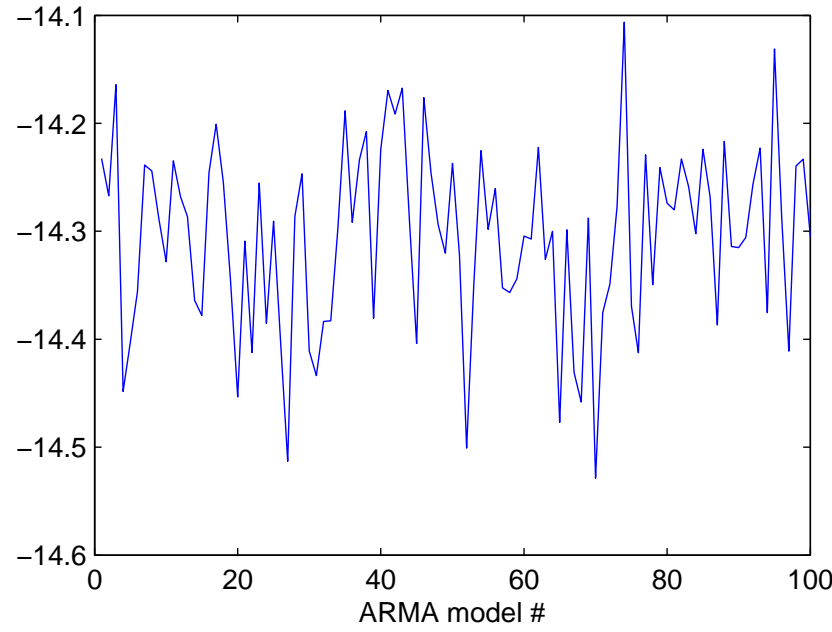

FIG. 5. AIC values for different ARMA models (Switched system).

\section{A. A numerical method for switched systems with time delay and white noise}

To perform numerical simulations we use Euler's scheme presented is Section III. However, the presence of the switching function implies that depending on the value of the random variable $\phi(t-\tau)$ the system is either governed by a stochastic differential equation, or a stochastic delay differential equation. We switch between these two systems when the random variable $\phi(t-\tau)$ is greater or smaller than the threshold value $\phi_{0}$. We assume the width of the dead zone, which can be interpreted as the accuracy of sensing, to be equal to $|\phi|=0.02 \mathrm{rad}$, which is approximately one degree.

\section{B. ARMA fitting}

We found that, similarly as in the continuous time case, ARMA model of order $(2,2)$ fits the position data. A hundred runs of the switched time system were generated. Then, one hundred ARMA models were determined, and the time series data were generated to obtain equivalent ARMA processes for each time series. Fig. 5 shows the AIC values for all the ARMA models.

As we have already discussed in Sec. III C, to produce time series data of an ARMA model system, it is required to estimate the variance of the underlying white noise process that drives the model. The standard deviation of the noise process was found to be $\sigma=0.000788$. The coefficients listed in Tab. II correspond to the average ARMA model coefficients of order $(2,2)$ obtained from the one hundred ARMA models, with the corresponding standard deviation for each set. We also included a representative run for a single output.
TABLE II. ARMA coefficients (for the switched stochastic system).

\begin{tabular}{cccccc}
\hline \hline & $\alpha_{1}$ & $\alpha_{2}$ & $\beta_{1}$ & $\beta_{2}$ & Fit \% \\
\hline position (average) & 1.7284 & -0.7569 & 1.0523 & 0.3983 & 95.34 \\
\hline velocity (average) & 1.341 & -0.525 & 0.300 & -0.021 & 67.62 \\
\hline position (single run) & 1.6777 & -0.8019 & 0.9637 & 0.3425 & 95.82 \\
\hline velocity (single run) & 1.2910 & -0.6413 & 0.2881 & -0.3834 & 67.93 \\
\hline Standard deviation & 0.0512 & 0.0519 & 0.0822 & 0.0638 & \\
\hline \hline
\end{tabular}

\section{Frequency spectrum}

Similarly as in the case of the continuous time system (23), in Fig. 6 we show a comparison between the position component of the time series data of the switched system (30) and (31) and its ARMA equivalent model, with the ARMA coefficients given in Tab. II (position component of a single run).

In Fig. 7 we show the Welch power spectrum of the position component of the switched system and its ARMA equivalent, in the case of a single run and by considering the averaged trajectory. In the case of a single run, in Fig. 7 we can see that these models shows a good fit in the low frequency band between 0.5 and 1.5 $\mathrm{Hz}$, while there is a growing difference in the power at higher frequency range.

For averaged spectral curves shown in Fig. 8, it can be seen that there is a slight difference in the power density at the lower frequency range, while there is a signification power difference at the higher frequency range. As we discussed earlier, this can be explained by the fact that it is the lower frequency band which is aimed to be fitted by means of ARMA. Finally, similarly as for the linear system, taking the averaged power spectrum has the effect of smoothing the spectral curve (compare Figures 7 and 8).

We now compute the matrix $C$ which gives the deterministic part of the continuous time representation of the ARMA model. The matrix $C$ (equation (19)) is found to be

$$
C=\left(\begin{array}{cc}
-11.487 & 11.671 \\
-8.601 & 8.435
\end{array}\right)
$$

We can verify that the eigenvalues of the matrix $C$ are equal to $\lambda_{1}=-1.525+1.078 i$ and $\lambda_{2}=-1.525-1.078 i$. Therefore, the switched stochastic system (30) and (31) corresponds to a second order linear oscillator with damped oscillatory motion driven by noise, similarly as in the continuous time case. 


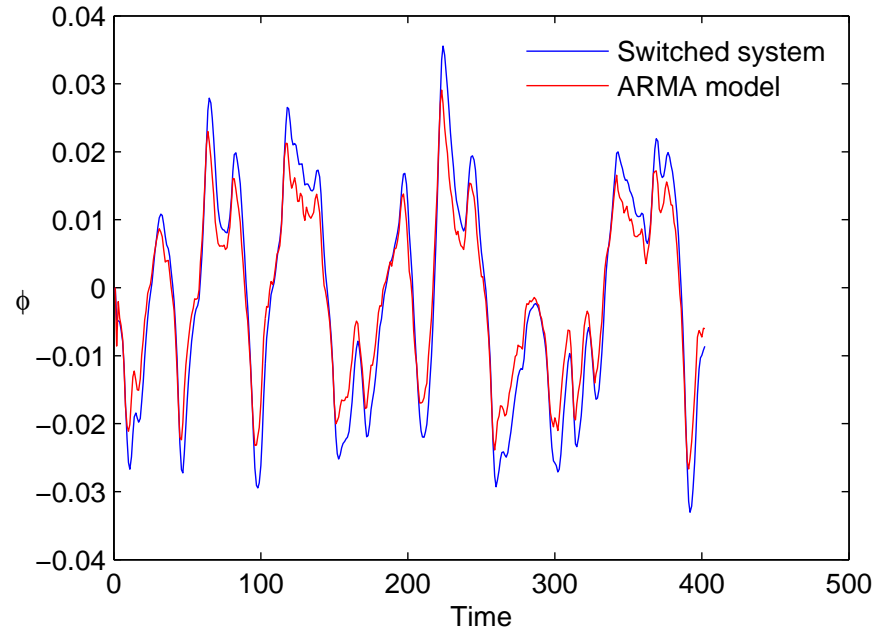

FIG. 6. Comparison between time series of the switched time system, and its equivalent ARMA model.

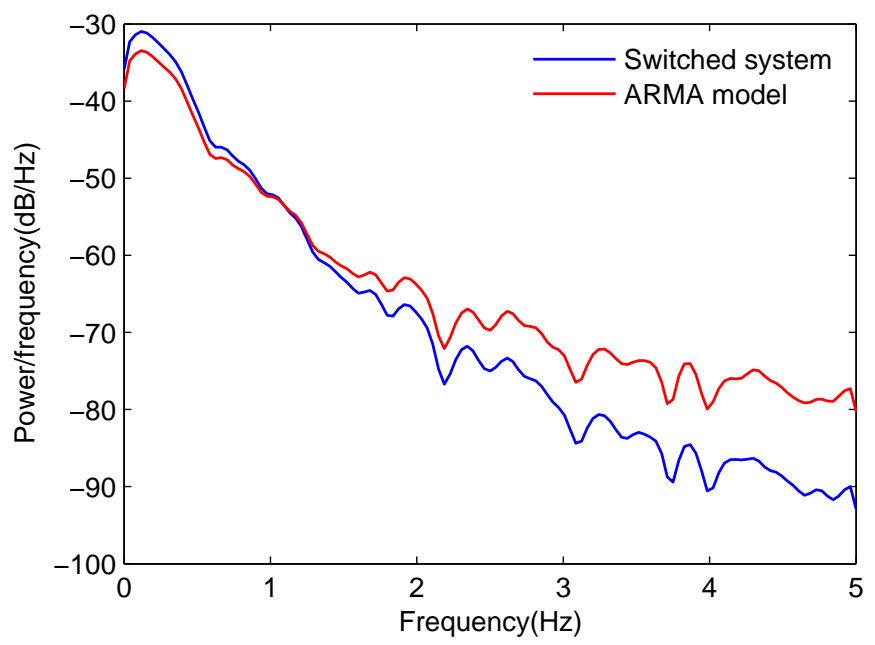

FIG. 7. Welch power spectral density estimate of the switched time system, and its equivalent ARMA model (single run).

\section{LINEAR VERSUS SWITCHED DYNAMICS - COMPARISON WITH EXPERIMENTAL POSTUROGRAPHIC DATA}

\section{A. Phase space - asymptotic evolutions}

For parameter values given in Sec. III B and IV A, we present the results of numerical simulations of the linear and switched systems in the absence of noise to understand the differences in the underlying dynamics. In the case of the linear system, the system evolution converges to a stable equilibrium (see Fig. 9(c)). On the other hand, in the case of the switched system, it is a pair of stable asymmetric limit cycles which capture

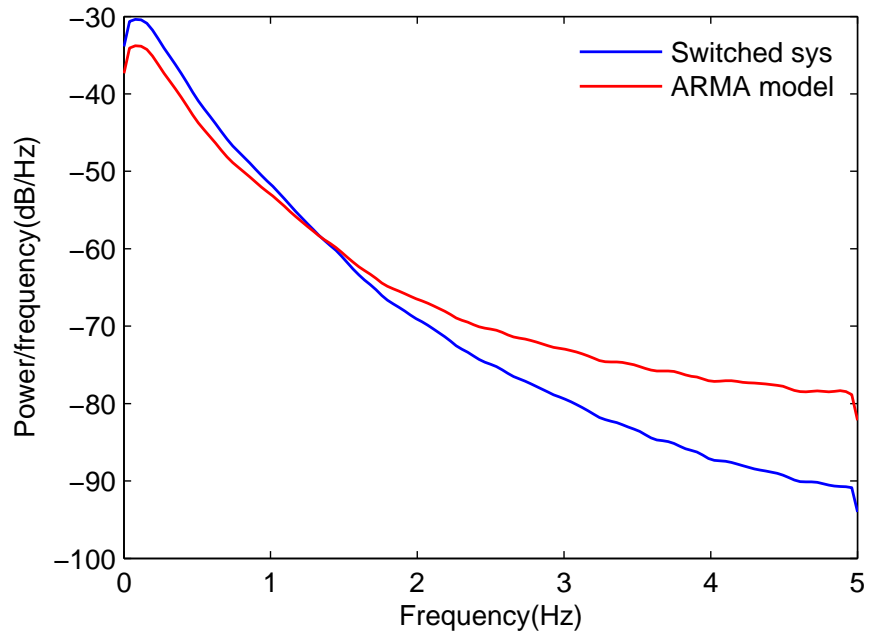

FIG. 8. Averaged power spectral density estimate of the switched time system, and its equivalent ARMA model.

the asymptotic dynamics; due to the system's symmetry, there is a pair of limit cycles in the system, as depicted in Fig. 9(a) and 9(b). Adding white noise to the switched system will cause the switchings of the evolution between neighbourhoods of these asymmetric limit cycles. Similar switching behaviour, induced by noise, was observed in ${ }^{14}$. An example of such an evolution is shown in Fig. 10(b). In the case of the linear system, the evolution will follow a random pattern of motion about the origin, as shown in Fig. 10(a). In Fig. 10(c), we present a phase plot obtained from experimental data of human quiet standing. From the phase plot, again we can not make any claims as to which system (the switched or the liner one) captures better the experimental posturographic data.

\section{B. Experimental posturographic data}

We consider the experiments of the position and velocity time series data of human subjects standing quietly with eyes closed or open. The data were collected at the IRM (Institute of Research into Human Movement) laboratory at Manchester Metropolitan University. Eight people, with no balance disorders, participated in the study. Each subject was standing quietly with eyes open for the duration of 240 s. Horizontal and vertical ground reaction forces were measured using a force plate with four strain gauge sensors (OR6-7; AMTI, Watertown, MA). The position of the center of pressure of the forward-backward and side-to-side body sway was calculated from the ground reaction forces. The time series data was recorded and sampled at $1 \mathrm{kHz}$. The experiment was then repeated with eyes closed. For the purposes of analysis, we expressed the force signal as angular position and velocity data. The signal was then filtered using lowpass 

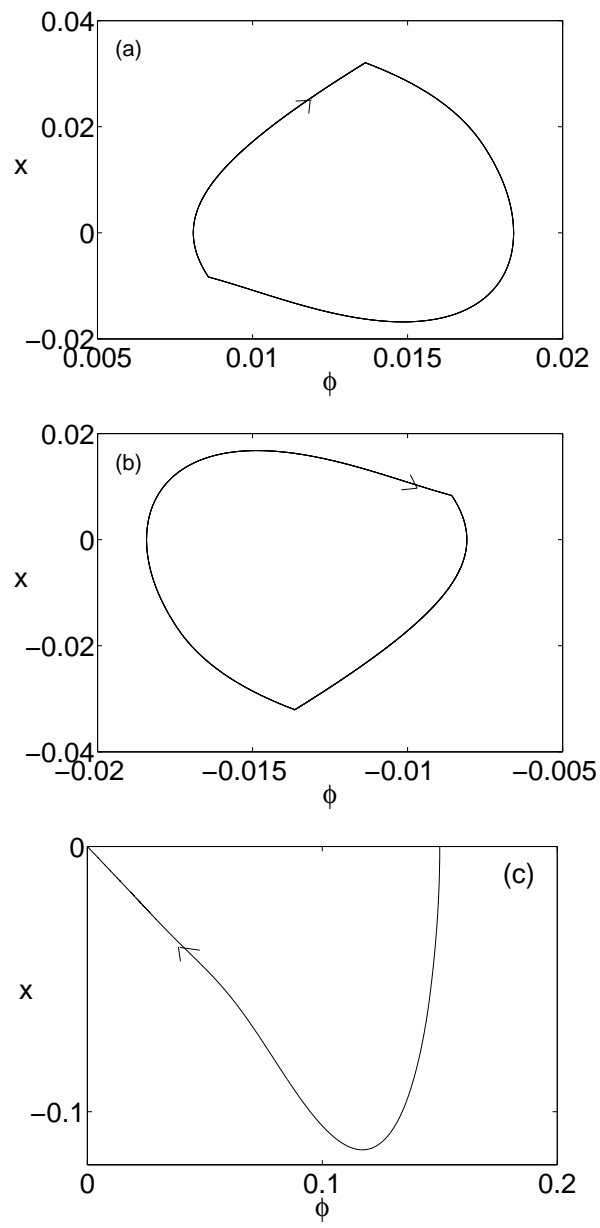

FIG. 9. A pair of stable asymmetric limit cycles, shown in (a) and (b), are present in the switched system (30) and (31), and in (c) we depict a convergence of the linear system (23) to its stable equilibrium point at the origin.

butterworth filter with the cut off frequency of $4 \mathrm{~Hz}$. For our purposes we used 40s snapshots of the data and every hundredth data point was used.

In Fig. 11 we present a comparison between the power spectrum of the continuous time system (23), switched stochastic system (30) and (31), and three posturographic data sets. It can be seen that the different systems have similar spectral properties over the entire frequency range. In Fig. 12 we also show a comparison between the power spectra generated by the equivalent ARMA models for all the systems. The agreement is not as good as when the power spectra of the original signals are compared and we are not able to make a justifiable claim as to which ARMA model system (corresponding to a linear or a switched model) fits better the ARMA models derived from posturographic data.
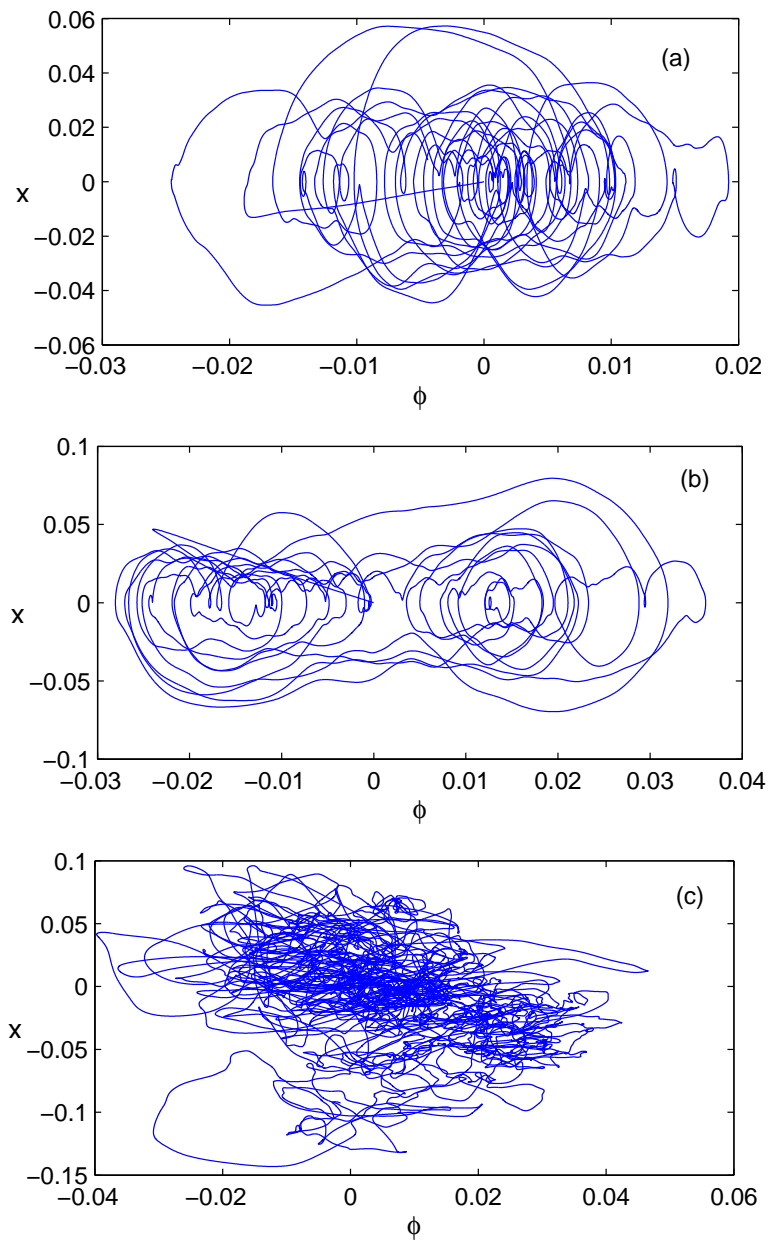

FIG. 10. In (a) we depict a representative example of an evolution of the linear system (23). In (b) we show a representative example of an evolution of the switched stochastic system (30) and (31), and (c) shows the phase space plot obtained from the experimental data of the movement during quiet standing.

\section{CONCLUSIONS}

In the paper, we numerically investigate a switched (characterised by a threshold) and a linear model of human balance control during quiet standing. We show that both systems can be modelled using second order ARMA models. To determine if there is any obvious qualitative effect of the presence of switched discontinuity on the ARMA model of the switched system, in comparison to the ARMA model of the linear system, we compare Welsh power spectral densities of the two systems with power spectral densities of their ARMA models. In both cases, that is when we compare the power spectrum of the switched system with the power spectrum of its ARMA model as well as when we compare the power spectrum of the linear system with the power spectrum of its ARMA model, the power spectra show a good fit in the lower frequency range (up 


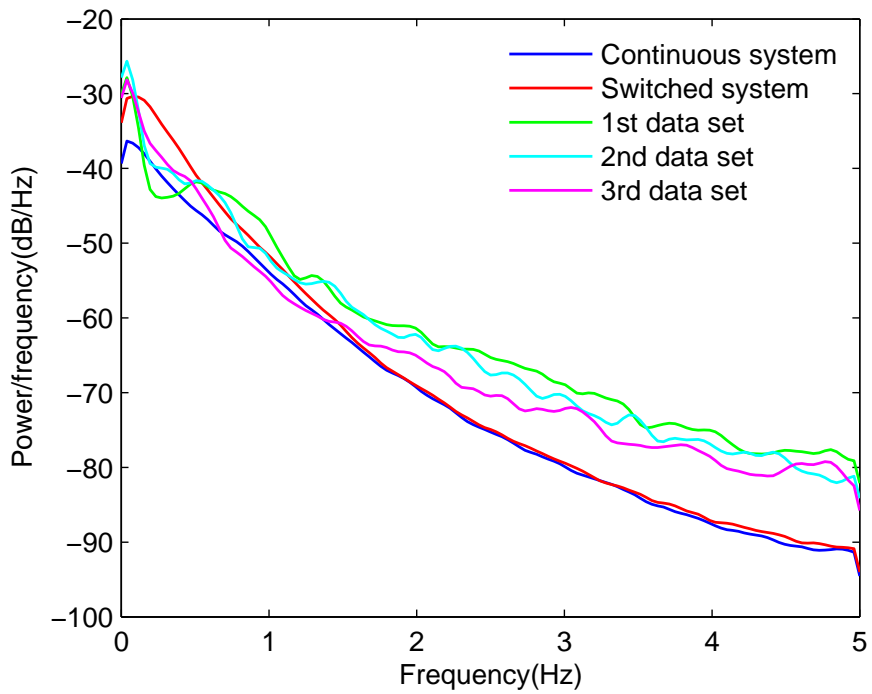

FIG. 11. Comparison between the power spectra of the continuous time system, switched system, and three posturographic data sets.

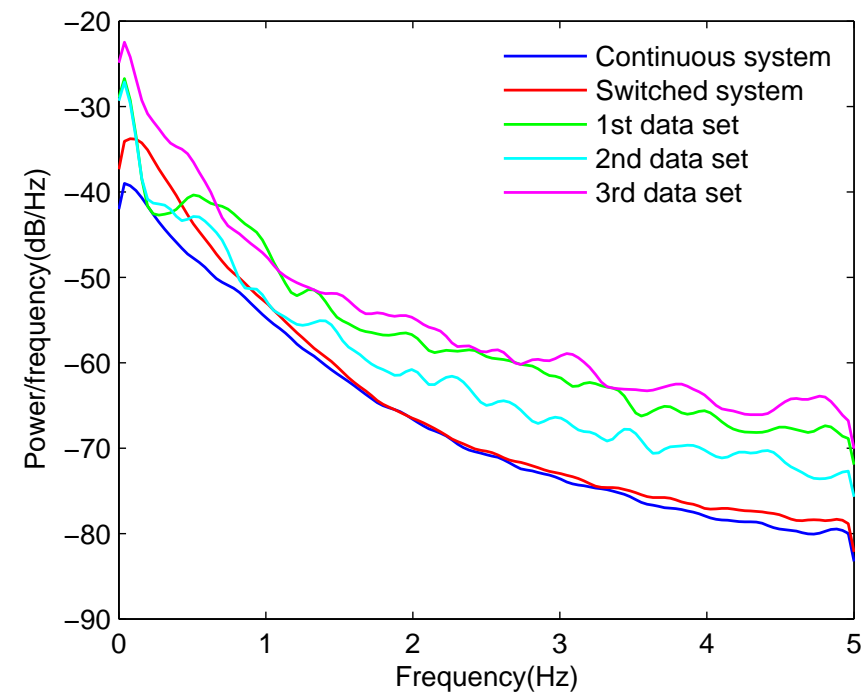

FIG. 12. Comparison between the power spectrum of the ARMA equivalents models of the continuous time system, switched time system, and three posturographic data sets.

to around $2 \mathrm{~Hz}$ ) with a mismatch for higher frequencies. This agrees with the theory because low order ARMA models match low frequency bands. Moreover, there are no obvious qualitative differences in both cases. We then compare power spectra of three ARMA models of three representative experimental posturographic data sets with ARMA models of the switched and linear systems. A reasonably good fit, to both models, is observed in the lower frequency range (up to $1.5 \mathrm{~Hz}$ ) with a growing mismatch for larger frequencies. Thus, AMRA model fitting may lead to misinterpretation of the results; that is, a good fit of a time series data with an ARMA model does not imply that the underlying process is linear and time invariant.

We also investigated the dynamics of underlying models in the absence of noise. We found qualitative differences in the asymptotic dynamics of both systems for parameter values used in our investigations; the dynamics of the linear system represent the fluctuations of a noisy equilibrium, whereas the dynamics of the switched systems are dominated by the effects of noisy bistable system formed by two co-existent limit cycle oscillators.

The work presented here suggests that further investigations are required to resolve the current controversy whether human balance control during quiet standing can be better described by a linear continuous time process or a process which contains discontinuities and/or open loops. Our research also raises questions of establishing mutual correlations between deterministic dynamics and noise and their effect on sway motion during quiet standing.

Acknowledgment Research funded by Engineering and Physical Sciences Research Council, grant number EP/K001353/1, and Manchester Metropolitan University.

\section{References}

${ }^{1}$ Craik, K. Theory of the human operator in control systems: I. The operator as an engineering system. British Journal of Psychology. 1947; Gen Sect 38:56-61.

2 Jeka, J.J., Oie, K.S., Kiemel, T. Multisensory information for human postural control: integrating touch and vision. Experimental Brain Research. 2000; 134:107-125.

${ }^{3}$ Johansson, R., Magnusson, M., Akesson, M. Identification of human postural dynamics. IEEE Transactions of Biomedical Engineering. 1988; 35:858-869.

${ }^{4}$ Winter, D.A. Biomechanics and Motor Control of Human Movement. New-York:Wiley; 1990.

${ }^{5}$ Winter, D.A., Patla, A.E., Prince, F., Isha,C. M., Gielo-Perczak, K. Stiffness control of balance in quiet standing. Journal of Neurophysiology. 1998; 80:1211-1221.

${ }^{6}$ Winter, D.A., Patla, A.E., Riedtyk, A.E., Ishac, M. Ankle muscle stiffness in the control of balance during quiet standing. Journal of Neurophysiology. 2001; 85:2630-2633.

${ }^{7}$ Jeka, J., Kiemel, T., Creath, R., Horak, F., Peterka, R. Controlling Human Upright Posture: Velocity Information Is More Accurate Than Position or Acceleration. Journal of Neurophysiology. 2004; 92:2368 - 2379.

${ }^{8}$ Kiemel, T., Oie, K.S., Jeka, J.J. Multisensory fusion and the stochastic structure of postural sway. Biological Cybernetics. 2002; 87:262-277.

${ }^{9} \mathrm{Yu}, \mathrm{X}$., Lewis, E. Studies with spike initiators: linearization by noise allows continuous signal modulation in neural networks. IEEE Trans Biomed Eng. 1989;36:36-43.

${ }^{10}$ Peterka, R.J., Loughlin, P.J. Dynamic Regulation of Sensorimotor Integration in Human Postural Control. Journal of Neurophysiology. 2004; 91:410 - 423 . 
${ }^{11}$ Kiemel T., Oie K.S., Jeka J.J. Slow Dynamics of Postural Sway Are in the Feedback Loop. J Neurophysiol. 2006; 95:1410-1418.

${ }^{12}$ Kowalczyk, P., Glendinning, P., Brown, M., Medrano-Cerda, G., Dallali, H., Shapiro, J. Modelling human balance using switched systems with linear feedback control. Journal of the Royal Society Interface. 2011; 9:234-245.

${ }^{13}$ Insperger, T., Milton, J., Stepan, G. Acceleration feedback improves balancing against reflex delay. Journal of the Royal Society Interface. 2012; 10:1098-0763.

${ }^{14}$ Milton, J., Townsend, J.L., King, M.A., Ohira, T. Balancing with positive feedback: the case for discontinuous control Philos. Trans A Math. Phys. Eng. Sci. 2009; 367:1181-1193.

${ }^{15}$ Bottaro, A., Casadio, M., Morasso, P., Sanguineti, V. Body sway during quiet standing: Is it theresidual chattering of an intermittent stabilization process? Human Movement Science. $2005 ; 24: 588615$.

${ }^{16}$ Gawthorp, P., Loram, I., Lakie, M., Gollee, H. Intermittent control: a computational theory of human control. Biological Cybernetics. 2011; 104:31-51.

${ }^{17}$ Loram, I.D., van de Kamp, C., Gollee, H., Gawthrop, P.J. Identification of intermittent control in man and machine. Journal of the Royal Society Interface. $2012 ; 10.1098 /$ rsif.2012.0142.

${ }^{18}$ Newell, K., Slobounov, S., Slobounova, E., Molenaar, P. Stochastic process in postural center-of-preasure profiles. Experimental Brain Research. 1997; 113:158-164.

${ }^{19}$ Eurich, C., Milton, J. Noise-induced transitions in human postural sway. Physical Review E. 1996; 54(6):6681-6684.

${ }^{20}$ Cabrera, J.L., Milton J.G. Stick balancing, falls and DragonKings. Phys. J. Special Topics E. 2012; 205:231-241.
${ }^{21}$ Bottaro, A., Yasutake, Y., Nomura, T., Casadio, M., Morasso, P. Bounded stability of the quiet standing posture:An intermittent control model. Human Movement Science. 2008; 27:473495.

${ }^{22}$ Yamada, N. Chaotic swaying of the upright posture. Human Movement Science. 1995; 14:711-726.

${ }^{23}$ Box, G., Jenkins, G., Reinsel, G. Time Series Analysis, Forecasting and Control. A JOHN WILEY AND SONS; 2008.

${ }^{24}$ Kloeden, P.E., Platen, E. Numerical Solution of Stochastic Differential Equations. Springer-Verlag; 1995.

${ }^{25}$ Frank, T., Beek, P. Stationary solutions of linear stochastic delay differential equations: Applications to biological systems. Physical Review E. 2001; 64:021917.

${ }^{26}$ Asai, Y., Tasaka, Y., Nomura, K., Nomura, T., Casadio, M., Morasso, P. A Model of Postural Control in Quiet Standing:Robust Compensation of Delay-Induced Instability Using IntermittentActivation of Feedback Control. PLoS ONE art no e6169. 2009; 4(7).

${ }^{27}$ Akaike, H. A new look at the statistical model identification. IEEE Transactions on Automatic Control. 1974; 19(6):716-723.

${ }^{28}$ Gammaitoni, L. Stochastic resonance and the dithering effect in threshold physical systems. Phys. Rev. E 52: 4691-4698, 1995.

${ }^{29}$ Hunter, J., Milton J., Ldtke H., Wilhelm B., Wilhelm H. Spontaneous fluctuations in pupil size are not triggered by lens accommodation. Vision Res. 2000; 40(5):567-73.

${ }^{30}$ Brockwell, P., Davis, R. Introduction to Time Series and Forecasting. Springer, New York; 1996.

${ }^{31}$ Welch, P. The Use of Fast Fourier Transform for the Estimation of Power Spectra: A Method Based on Time Averaging Over Short, Modified Periodograms. IEEE Transactions on Audio Electroacoustics. 1967;AU-15:70-73. 